\title{
Compact U-Slotted Antenna for Broadband Radar Applications
}

\author{
S. Costanzo and A. Costanzo \\ Dipartimento di Ingegneria Informatica, Modellistica, Elettronica e Sistemistica, Università della Calabria, 87036 Rende, Italy \\ Correspondence should be addressed to S. Costanzo; costanzo@deis.unical.it
}

Received 29 May 2013; Accepted 4 September 2013

Academic Editor: Alvaro Rocha

Copyright (C) 2013 S. Costanzo and A. Costanzo. This is an open access article distributed under the Creative Commons Attribution License, which permits unrestricted use, distribution, and reproduction in any medium, provided the original work is properly cited.

\begin{abstract}
The original U-shaped patch antenna is properly modified in this work to provide a compact and broadband antenna configuration with reduced cross-polar effects, well suitable for modern radar applications. The proposed antenna layout is applied to design, realize, and test two different prototypes working at P-band and C-band, typically adopted for ground-penetrating radar. The experimental results successfully demonstrate a large operating bandwidth between $15 \%$ and $20 \%$, a significant reduction of size (about half of the standard configuration), and a low cross-polarization level within the operating frequency range.
\end{abstract}

\section{Introduction}

The new generation radar systems require advanced components and materials $[1-4]$ in order to properly exhibit their innovative features $[5,6]$. For this motivation, in recent years, most of the classical hardware radar architecture functions have begun to be implemented via software, in order to reduce the costs and the complexity of the entire system [79]. Antennas often represent the most critical hardware component in the radar system, and the design of the radiating element needs to satisfy several constraints required by the novel applications, especially in terms of bandwidth [10-14] and quality of the radiation pattern, while guaranteeing, at the same time, a compact configuration. Microstrip patch antennas are largely employed in radar systems due to their low costs and easy manufacturing, low profile, light weight, and good strength, but the use of standard single-layer microstrip patch is not suitable for most radar applications, mainly due to its narrowband behavior. Additional resonant elements, such as slots, may be included in the radiating surface in order to enhance the bandwidth. The U-slot configuration is typically adopted at this aim, as the multiple resonances introduced by the slot allow it to produce a unique wideband response. Various examples exist in the literature for the standard U-slot patch antenna configuration. In [15], a compact U-slotted microstrip patch, performing a wideband behavior, has been presented, and in [16] an example of multiband U-slot antenna has been proposed. Even if all existing works show that U-slot configuration yields good results in terms of bandwidth and compactness, the additive resonances significantly disturb the main flow of the surface current, thus increasing the cross-polarization level. As a consequence of this, the useful radiation bandwidth of standard $\mathrm{U}$-slot configuration is significantly reduced with respect to that achieved in terms of return loss behavior, thus preventing its application in modern broadband radar systems.

Most of the existing works in the literature for the standard U-slot antenna are focused on the achievement of a tradeoff between bandwidth and size reduction but not giving an effective procedure for reducing the cross-polar component.

Starting from the study of U-slot antenna behavior presented in [17], the original configuration is properly modified in this work to guarantee the wideband radiation feature, while reducing, at the same time, the cross-polarization effects due to the complex combination of the antenna and the slot modes. In the modified U-slot layout, the current path forced by the slot is properly constrained to provide a low cross-polarization effect, while achieving a significant reduction in the total size of the radiating element. To prove the effectiveness of the proposed antenna configuration, experimental validations on two different prototypes, useful for P-band and C-band radar applications, are successfully discussed. In particular, radiation bandwidths between $15 \%$ and $20 \%$ are demonstrated with reduced cross-polar levels and compact dimensions. 


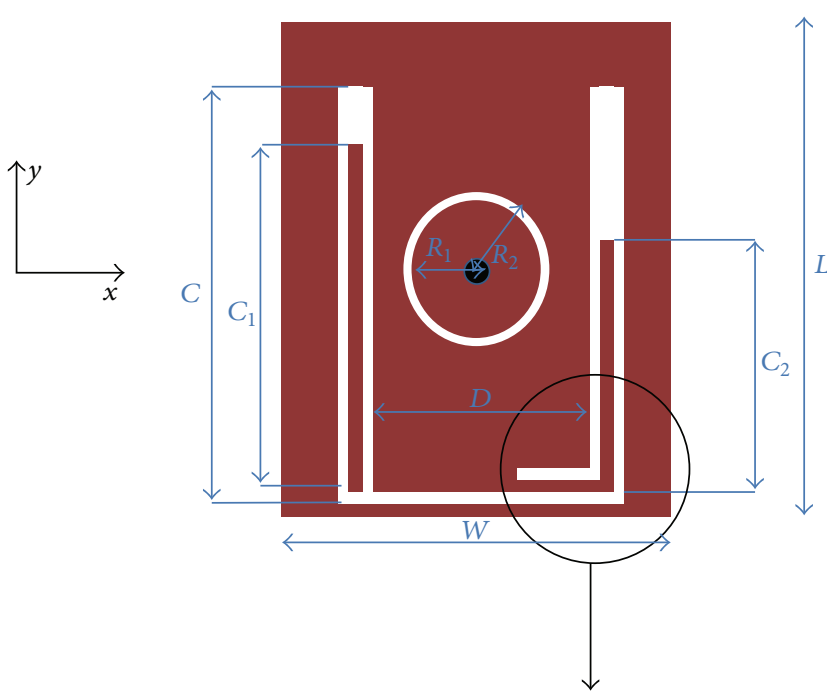

(a)

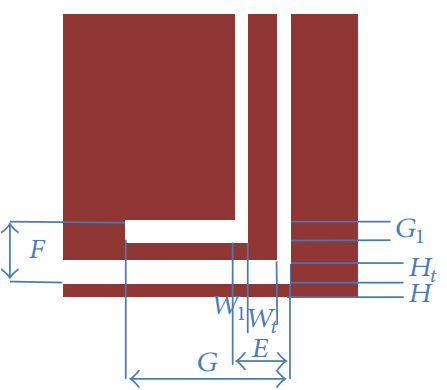

(b)

FIgURE 1: Layout of modified U-slot antenna: (a) top layer and (b) enlarged view of right corner.

\section{Antenna Configuration}

The technique based on the application of slot cuts in the radiating element is widely adopted in the literature to improve the bandwidth and/or reduce the antenna size [1820]. In this paper, the basic U-slot shape is considered, properly modifying the layout as in Figure 1, in order to control the undesired current giving cross-polarization, but guaranteeing at the same time a compact structure with a large bandwidth behavior. Two main differences exist between the standard configuration and the proposed design, namely,

(i) asymmetrical strips introduced in the arms of the vertical U-shaped slots;

(ii) a further modification to the horizontal arm of the slot in order to introduce more degrees of freedom in the design, thus better controlling the current flow and the antenna resonance frequencies.

In the example shown in Figure 1, the polarization is vertical, so the layout is designed in order to avoid $x$-directed modal propagation.

As in classical U-slot antenna, a low permittivity substrate is used to reduce the antenna $Q$ factor, thus increasing the bandwidth. In the proposed configuration, a ring slot is introduced around the central feeding point to compensate the large probe inductance due to the presence of the low permittivity substrate, which could worsen the impedance matching after a certain value of thickness. As discussed in [17], antenna and U-slot modes combinations, in the classical design, give undesired $x$-directed currents; hence, an unwanted increase of cross-polarization is produced. As a consequence of this, a particular ratio between the vertical and the horizontal arms needs to be considered to avoid this problem, but bandwidth requirements cannot always be satisfied according to this constraint.
The combination of the cuts in the proposed design allows a good tradeoff between bandwidth improvement and paths minimization of unwanted $x$-directed modes, avoiding a limiting constraint in the arms dimensions. The asymmetry in the vertical strip is necessary in order to achieve a better impedance matching, a significant size reduction, and a correct compensation of the $x$-directed currents. In the next section, the proposed antenna layout is successfully applied to design compact and wideband prototypes, with low crosspolarization effects, useful for P-band and C-band radar systems.

\section{P-Band and C-Band Antenna Prototypes}

Radar systems requiring a deep penetration into the monitoring scenario usually work in the relatively low region of the electromagnetic spectrum. For these applications, Pband operating frequencies are largely adopted. On the other hand, when high resolutions but lower waves penetrations are required, such as in short-range weather observation, C-band frequencies are usually considered.

In order to make the proposed antenna configuration well suitable for typical radar applications, two prototypes, respectively, working in P-band and C-band, are designed, realized, and tested. A low permittivity dielectric (foam, $\varepsilon_{r}=$ 1.07) simulating an air filling is assumed as main substrate, while an upper thin $(0.762 \mathrm{~mm})$ standard layer of Arlon DiClad870 $\left(\varepsilon_{r}=2.33\right)$ is uniquely employed for robustness reasons, to better support the modified U-slot patch antenna. A thickness approximately equal to $\lambda_{o} / 11$ is adopted in both cases for the foam substrate. An optimization procedure, based on the proper tuning of all geometrical parameters illustrated in Figure 1, is applied to derive, after a few iteration steps, the antennas dimensions, fully reported in Tables 1 and 2 , respectively, for the P-band and the C-band prototypes.

The adopted design procedure assumes the study presented in [17] as a starting point, improving the configuration, 


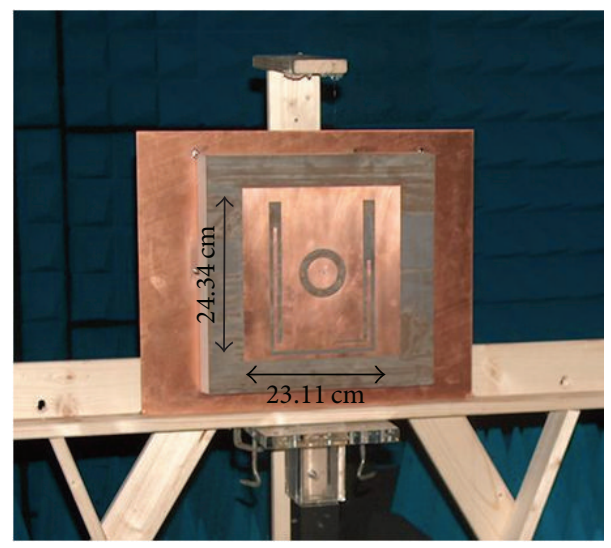

Figure 2: Photograph of P-band prototype into the anechoic chamber of Microwave Laboratory at University of Calabria.

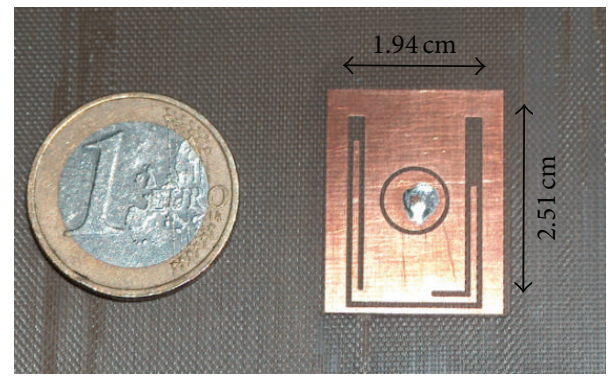

Figure 3: Photograph of C-band prototype.

in terms of compactness and wideband features, by assuming the following expressions for the patch width $W$, given as

$$
W=\frac{c}{3.55 f_{o}} \sqrt{\varepsilon_{r}}
$$

with $c$ being the free-space velocity.

The inner and outer radii $R_{1}, R_{2}$ of the ring slot are chosen on the basis of the design procedure outlined in [21].

Finally, the parameters $C_{1}, C_{2}$, relative to the modified $\mathrm{U}$ slot arms, are computed by the following expressions:

$$
\begin{gathered}
C_{1}=\left(\frac{c}{4 f_{o} \sqrt{\varepsilon_{r}}}-\frac{E}{2}\right) \cdot\left(1+\frac{D f_{o} \alpha}{c}\right), \\
C_{2}=\left(\frac{c}{4 f_{o} \sqrt{\varepsilon_{r}}}-\frac{E}{2}\right) \cdot\left(1-\frac{D f_{o}}{\alpha c}\right),
\end{gathered}
$$

where the term $\alpha$, assuming values in the range between 0.88 and 0.93 , is adopted as tuning parameter to optimize, after a few iteration steps (typically two or three), the radiating structure in terms of impedance matching, achieving a bandwidth between $15 \%$ and $20 \%$.

Both configurations reported in Tables 1 and 2 show a reduction of about $50 \%$ in the patch surface area, thus providing a significant compactness. As a matter of fact, a standard U-slot patch antenna [17] presents at P-band a width equal to $492.6 \mathrm{~mm}$, while the proposed prototype in Table 1 (Figure 2) has a strongly reduced width equal to $231.1 \mathrm{~mm}$. Similar features can be observed for the C-band prototype (Figure 3).
TABLE 1: Geometrical parameters of P-band prototype (centre frequency: $450 \mathrm{MHz}$ ).

\begin{tabular}{lccc}
\hline Parameter & Dimension $(\mathrm{mm})$ & Parameter & Dimension $(\mathrm{mm})$ \\
\hline$L$ & 243.4 & $R_{1}$ & 22 \\
$W$ & 231.1 & $F$ & 18 \\
$D$ & 156 & $W_{t}$ & 6 \\
$E$ & 18 & $G$ & 59.2 \\
$C$ & 218 & $R_{2}$ & 35 \\
$C_{1}$ & 181.5 & $W_{1}$ & 6 \\
$H$ & 6 & $G_{1}$ & 5 \\
$C_{2}$ & 231.1 & $H_{t}$ & 5 \\
\hline
\end{tabular}

TABLE 2: Geometrical parameters of C-band prototype (centre frequency: $4.25 \mathrm{GHz}$ ).

\begin{tabular}{lccc}
\hline Parameter & Dimension $(\mathrm{mm})$ & Parameter & Dimension $(\mathrm{mm})$ \\
\hline$L$ & 25.1 & $R_{1}$ & 3.4 \\
$W$ & 19.4 & $F$ & 1.9 \\
$D$ & 11.0 & $W_{t}$ & 0.6 \\
$E$ & 1.9 & $G$ & 5.5 \\
$C$ & 21.5 & $R_{2}$ & 3.9 \\
$C_{1}$ & 18.0 & $W_{1}$ & 0.6 \\
$H$ & 0.6 & $G_{1}$ & 0.6 \\
$C_{2}$ & 13.0 & $H_{t}$ & 0.6 \\
\hline
\end{tabular}

Experimental validations of prototypes in Figures 2 and 3 are performed into the anechoic chamber of the Microwave Laboratory at University of Calabria, equipped for both nearfield [22-24] and far-field measurements.

For the P-band prototype, a bandwidth of about 15\% is successfully demonstrated by the measured return loss behavior reported in Figure 4.

The operation bandwidth is further confirmed in terms of radiation features by the measured $\mathrm{H}$-plane patterns illustrated in Figure 5 at different frequencies. As a matter of fact, it is straightforward to observe that the crosspolar component properly remains below the copolar field within the entire frequency band. This advantageous result is achieved by accurately minimizing the $x$-directed currents 


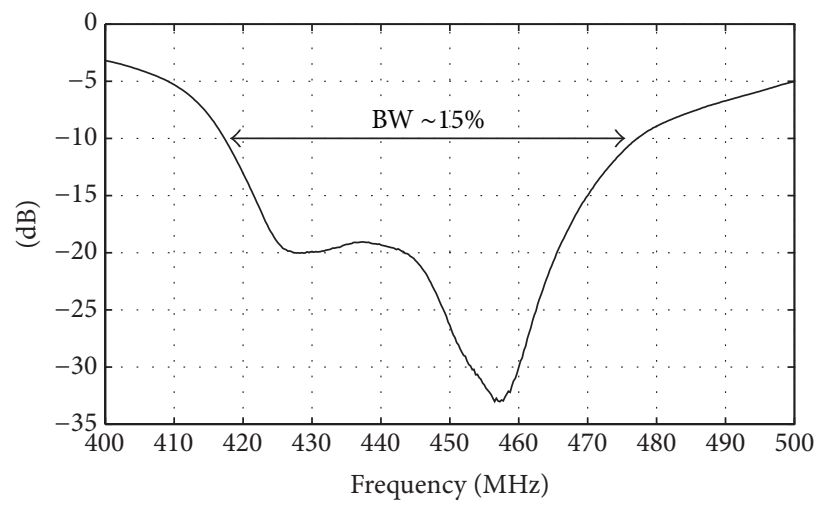

FiguRe 4: Measured return loss of P-band antenna prototype.

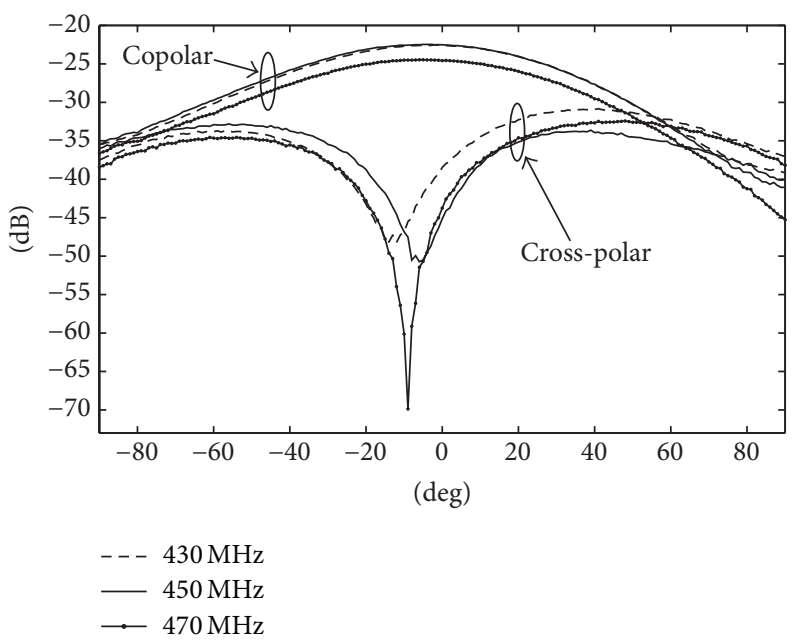

FIGURE 5: Measured radiation patterns of P-band antenna prototype at different frequencies.
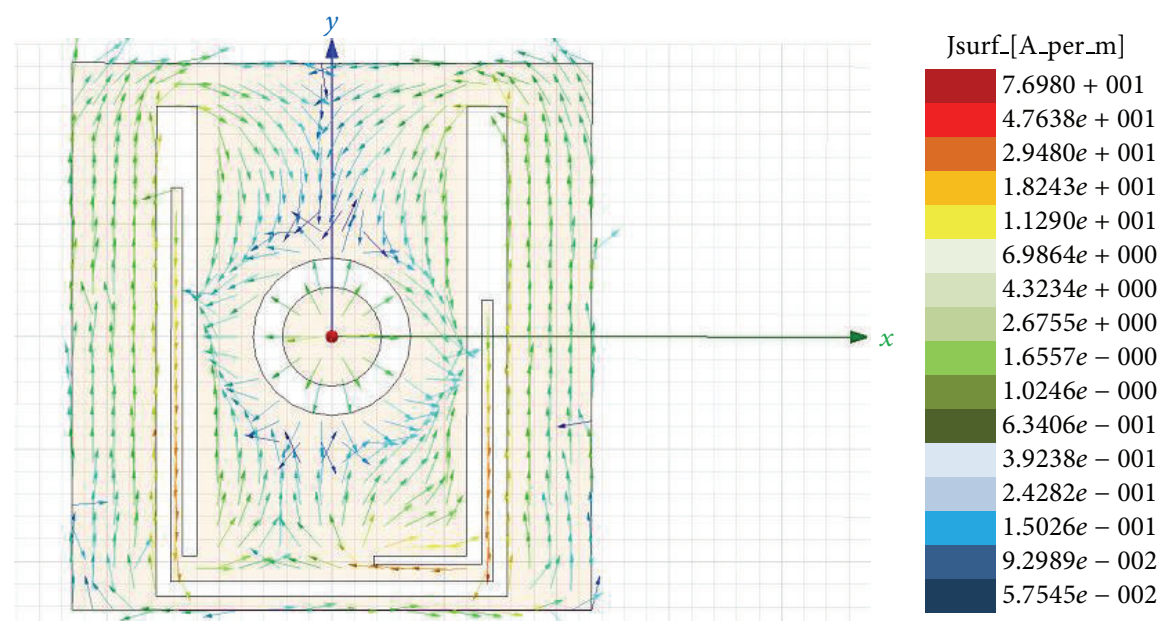

FIGURE 6: Simulated current distribution on the proposed P-band antenna prototype.

on the radiating elements, which are mainly dependent on the geometrical parameters $D, C, G_{1}$, and $G_{2}$ (Figure 1).

To demonstrate the proper reduction of the horizontal component of the current, a plot of the simulated current distribution on the P-band prototype at the central operating frequency of $450 \mathrm{MHz}$ is reported in Figure 6. A strongly $y$-directed current can be observed in the strips inside the vertical arms of the U-slot, while no relevant $x$-directed current can be identified in the region outside the slot.

For comparison purpose, the simulated current distribution on a standard U-slot antenna [17] working at the same frequency is reported in Figure 7. In this case, a relevant 

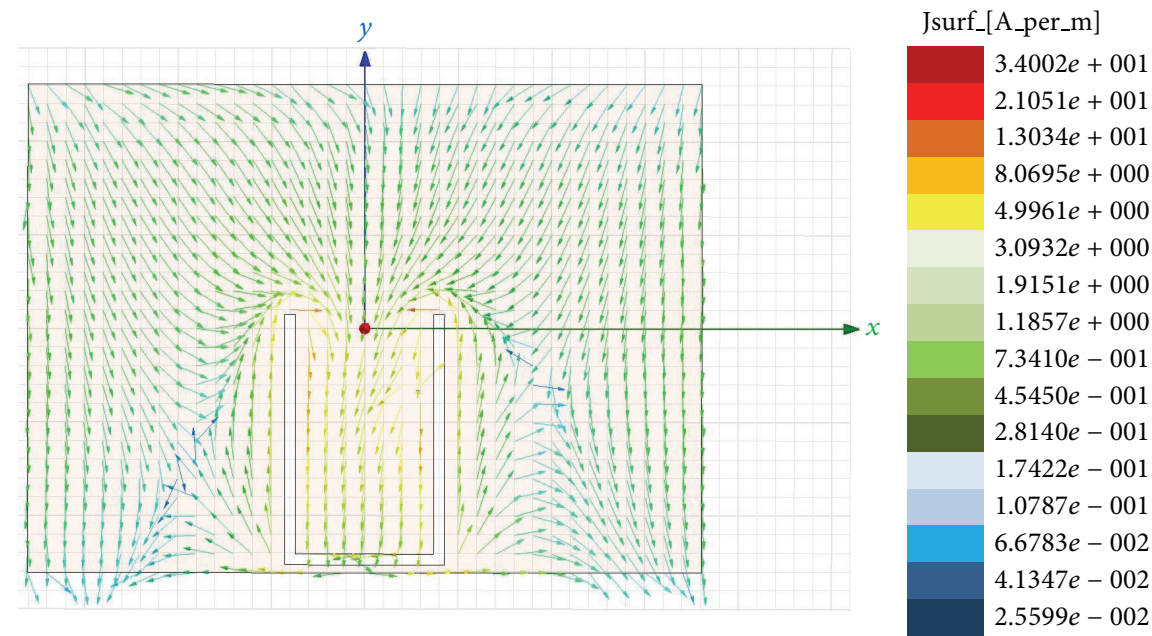

FIGURE 7: Simulated current distribution on a standard P-band U-slot antenna.

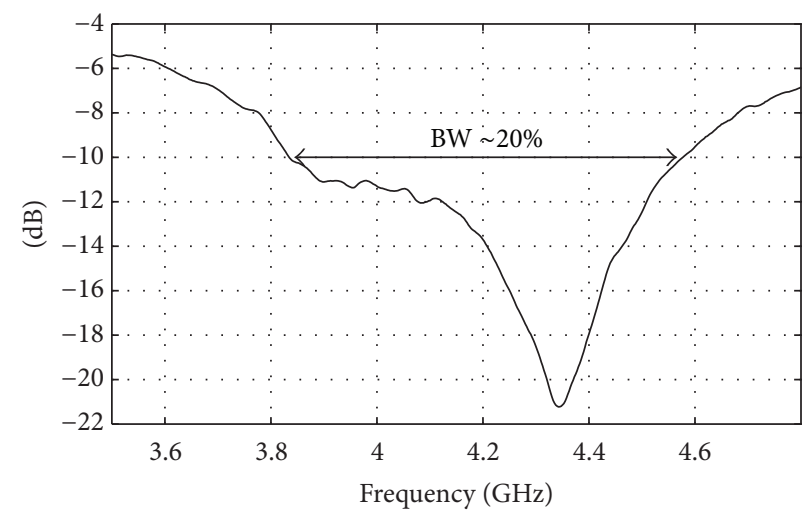

FIGURE 8: Measured return loss of C-band antenna prototype.

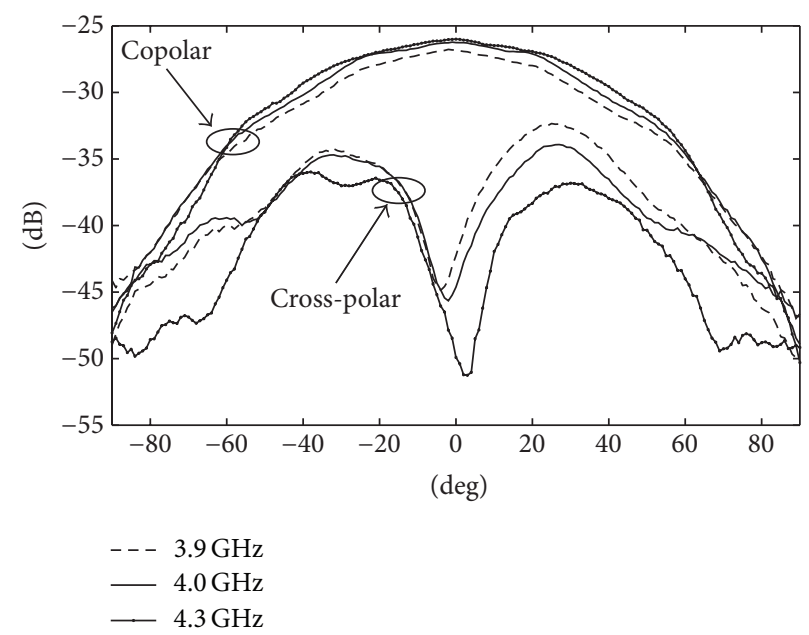

FIGURE 9: Measured radiation patterns of C-band antenna prototype at different frequencies.

$x$-directed current circulation can be observed, thus causing a more relevant cross-polar level with respect to the configuration proposed in this work.

Analogous experimental validations are performed on the C-band prototype (Figure 3), for which the measured return loss shows an operating bandwidth of about $20 \%$
(Figure 8). This is again confirmed by the measured radiation patterns of Figure 9, where the cross-polar fields are properly maintained below the copolar components when approaching the bandwidth extremes, thus overcoming the main limitation in terms of high cross-polarization effects, observed in [17] for the standard U-slot patch configuration. 


\section{Conclusion}

An innovative U-shaped slot antenna has been discussed in this work to significantly overcome the limitations in terms of high cross-polar fields for the standard U-slot patch. The proposed slot design technique is able to provide a compact structure with a large operating bandwidth, without occurring in undesired cross-polarization effects strongly affecting the useful radiation frequency range. An effective broadband bandwidth between $15 \%$ and $20 \%$ has been successfully demonstrated by experimental validations on P-band and Cband prototypes, useful to be adopted as radiating elements into penetrating radar systems. As a matter of fact, the achieved broadband behavior assumes a great relevance at the radar system level, where wideband antennas are strongly necessary to support more demanding modes of operation, often condensing the received echo information into more channels, so as to enhance the radar capabilities in terms of target classification.

\section{Acknowledgments}

The authors would like to thank Mr. Ennio Marozzo for the precious technical support in the realization of the antenna prototypes. This work has been carried out under the framework of PON 01_01503 National Italian Project "Landslides Early Warning," financed by the Italian Ministry of University and Research.

\section{References}

[1] S. Costanzo, I. Venneri, G. D. Massa, and A. Borgia, "Benzocyclobutene as substrate material for planar millimeter-wave structures: dielectric characterization and application," Journal of Infrared, Millimeter, and Terahertz Waves, vol. 31, no. 1, pp. 66-77, 2010.

[2] S. Costanzo, F. Venneri, A. Borgia, I. Venneri, and G. di Massa, " $60 \mathrm{GHz}$ microstrip reflectarray on a benzocyclobutene dielectric substrate," IET Science, Measurement and Technology, vol. 5, no. 4, pp. 134-139, 2011.

[3] S. Costanzo, A. Borgia, I. Venneri, and G. di Massa, "Millimeterwaves structures on benzocyclobutene dielectric substrate," Radioengineering, vol. 20, no. 4, pp. 785-789, 2011.

[4] G. di Massa, S. Costanzo, and O. H. Moreno, "Accurate circuit model of open resonator system for dielectric material characterization," Journal of Electromagnetic Waves and Applications, vol. 26, no. 5-6, pp. 783-794, 2012.

[5] F. Venneri, S. Costanzo, and G. di Massa, "Tunable reflectarray cell for wide angle beam-steering radar applications," Journal of Electrical and Computer Engineering, vol. 2013, Article ID 325746, 7 pages, 2013.

[6] F. Venneri, S. Costanzo, and G. di Massa, "Design of a reconfigurable reflectarray unit cell for wide angle beam-steering radar applications," Advances in Intelligent Systems and Computing, vol. 206, pp. 1007-1013, 2013.

[7] S. Costanzo, F. Spadafora, A. Borgia, O. H. Moreno, A. Costanzo, and G. di Massa, "High resolution software defined radar system for target detection," Advances in Intelligent Systems and Computing, vol. 206, pp. 997-1005, 2013.
[8] S. Costanzo, F. Spadafora, O. H. Moreno, F. Scarcella, and G. di Massa, "Multiband software defined radar for soil discontinuities detection," Journal of Electrical and Computer Engineering, vol. 2013, Article ID 379832, 6 pages, 2013.

[9] S. Costanzo, F. Spadafora, G. di Massa et al., "Potentialities of USRP-based software defined radar systems," Progress in Electromagnetics Research B, vol. 53, pp. 417-435, 2013.

[10] S. Costanzo, F. Venneri, and G. di Massa, "Bandwidth enhancement of aperture-coupled reflectarrays," Electronics Letters, vol. 42, no. 23, pp. 1320-1322, 2006.

[11] S. Costanzo, G. di Massa, I. Venneri, and G. Arriendola, "Hybrid array antenna for broadband millimeter-wave applications," Progress in Electromagnetics Research, vol. 83, pp. 173-183, 2008.

[12] F. Venneri, S. Costanzo, G. di Massa, and G. Amendola, "Aperture-coupled reflectarrays with enhanced bandwidth features," Journal of Electromagnetic Waves and Applications, vol. 22, no. 11-12, pp. 1527-1537, 2008.

[13] S. Costanzo, I. Venneri, and G. di Massa, "Wideband hybrid array concept for millimeter-wave applications," Journal of Infrared, Millimeter, and Terahertz Waves, vol. 30, no. 4, pp. 374380, 2009.

[14] F. Venneri, S. Costanzo, and G. di Massa, "Bandwidth behavior of closely spaced aperture-coupled reflectarrays," International Journal of Antennas and Propagation, vol. 2012, Article ID 846017, 11 pages, 2012.

[15] G. F. Khodaei, G. Nourinia, and C. Ghobadi, "A practical miniaturized U-slot patch antenna with enhanced bandwidth," Progress in Electromagnetics Research B, vol. 3, pp. 47-62, 2008.

[16] K. F. Lee, K. M. Luk, K. M. Mak, and S. L. S. Yang, "On the use of U-slots in the design of dual-and triple-band patch antennas," IEEE Antennas and Propagation Magazine, vol. 53, no. 3, pp. 60$74,2011$.

[17] S. Weigand, G. H. Huff, K. H. Pan, and J. T. Bernhard, "Analysis and design of broad-band single-layer rectangular Uslot microstrip patch antennas," IEEE Transactions on Antennas and Propagation, vol. 51, no. 3, pp. 457-468, 2003.

[18] F. Jolani and A. M. Dadgarpour, "Compact M-slot folded antenna for WLAN," Progress in Electromagnetics Research Letters, vol. 3, pp. 35-42, 2008.

[19] J. A. Ansari, P. Singh, S. K. Dubey, R. U. Khan, and B. R. Vishvakarma, "H-shaped stacked patch for dual-band operation," Progress in Electromagnetics Research, vol. B5, pp. 291-302, 2008.

[20] F. Yang, X. X. Zhang, X. Ye, and Y. Rahmat-Samii, "Wide-band E-shaped patch antennas for wireless communications," IEEE Transactions on Antennas and Propagation, vol. 49, no. 7, pp. 1094-1100, 2001.

[21] P. S. Hall, "Probe compensation in thick microstrip patches," Electronics Letters, vol. 23, no. 11, pp. 606-607, 1987.

[22] S. Costanzo and G. di Massa, "Far-field reconstruction from phaseless near-field data on a cylindrical helix," Journal of Electromagnetic Waves and Applications, vol. 18, no. 8, pp. 10571071, 2004.

[23] S. Costanzo and G. di Massa, "Direct far-field computation from bi-polar near-field samples," Journal of Electromagnetic Waves and Applications, vol. 20, no. 9, pp. 1137-1148, 2006.

[24] S. Costanzo and G. di Massa, "Near-field to far-field transformation with planar spiral scanning," Progress in Electromagnetics Research, vol. 73, pp. 49-59, 2007. 

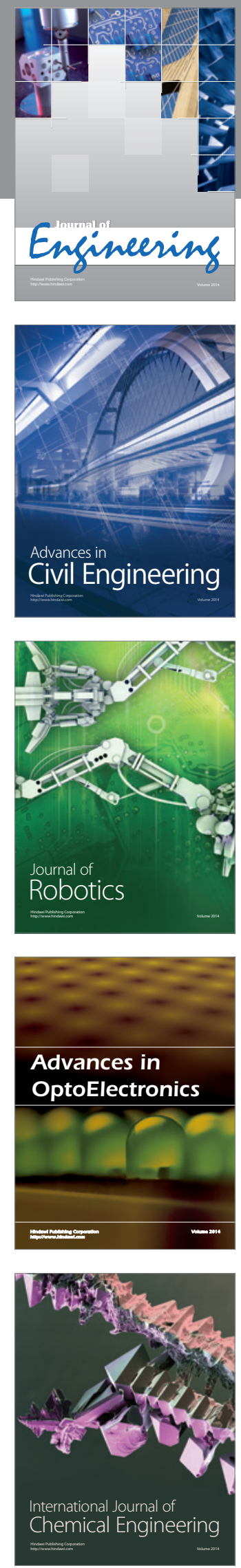

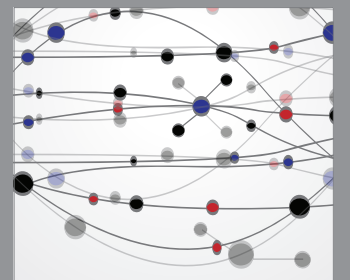

The Scientific World Journal
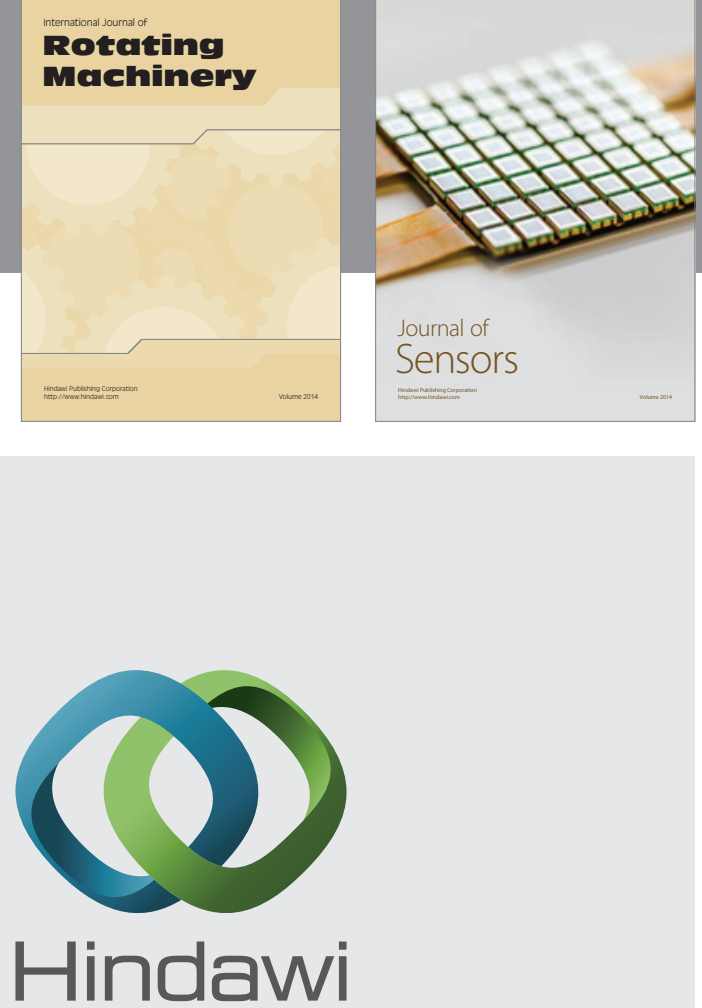

Submit your manuscripts at http://www.hindawi.com
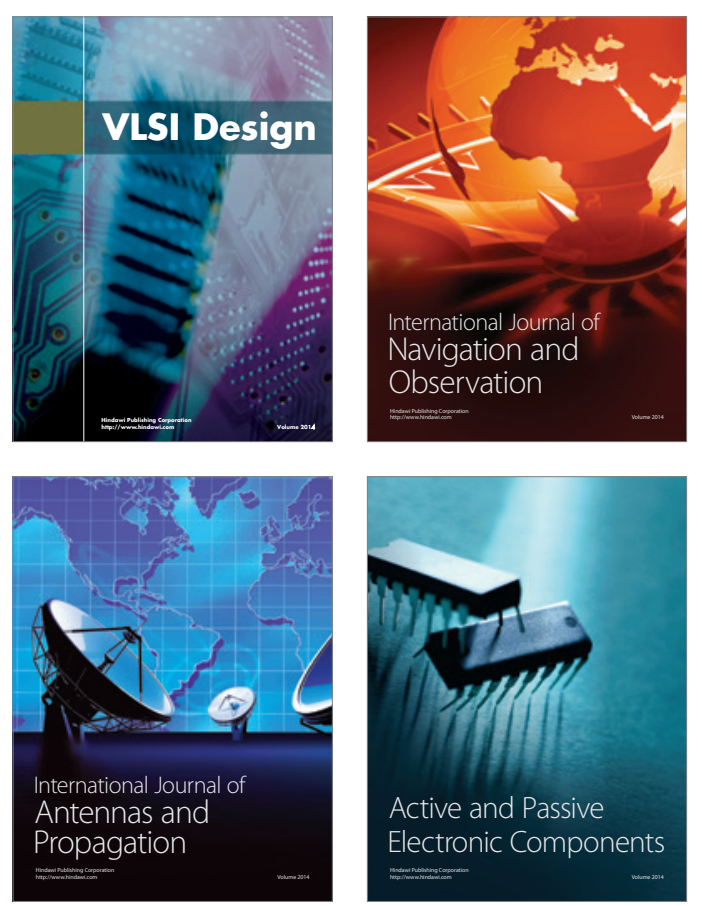
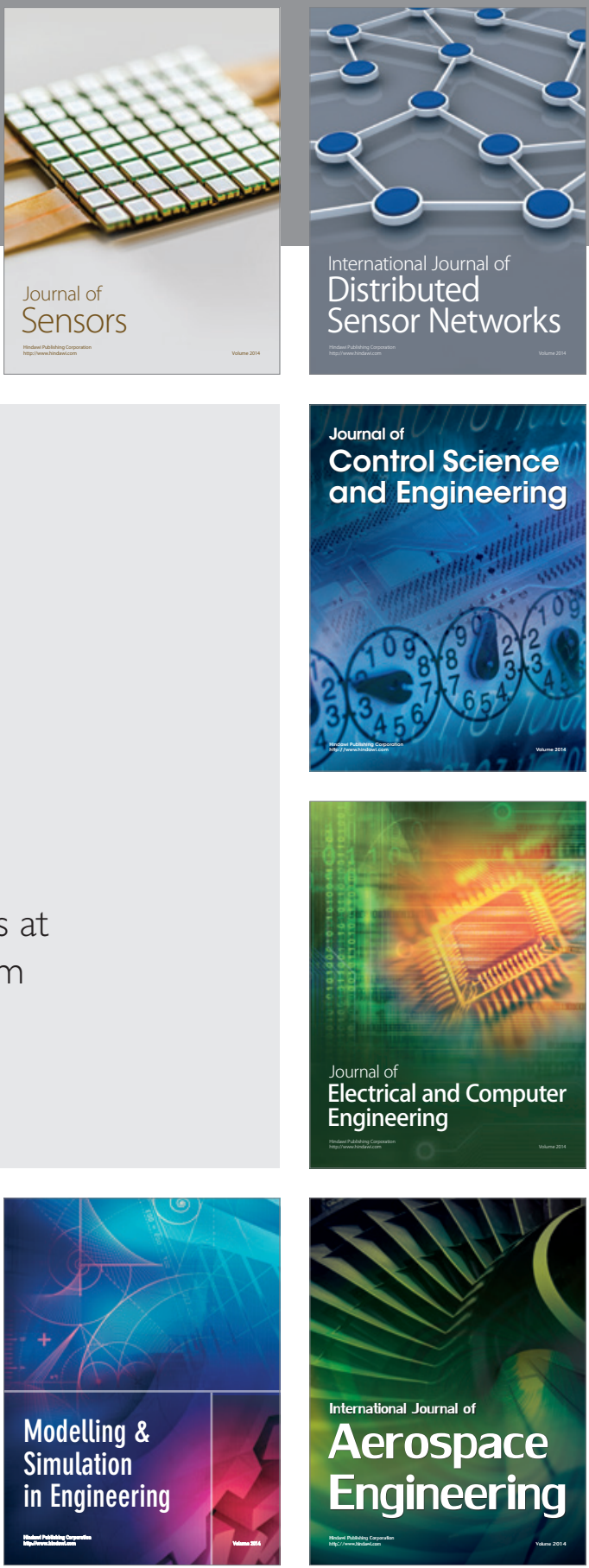

Journal of

Control Science

and Engineering
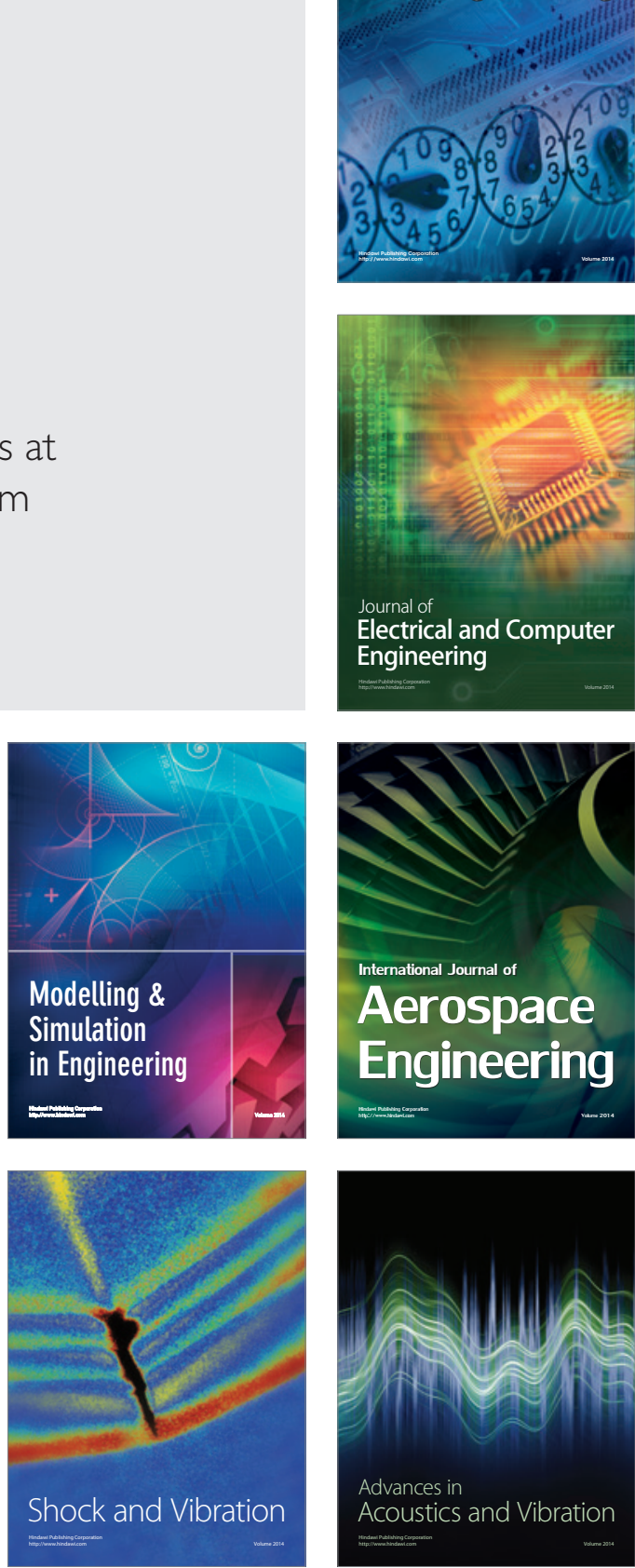